\title{
Table Height Position
}

National Cancer Institute

\section{Source}

National Cancer Institute. Table Height Position. NCI Thesaurus. Code C69238.

The elevation of a table in relation to a reference point. 